\title{
Partial Symmetries for Nonlinear Systems
}

\author{
H. Nijmeijer and A. J. van der Schaft \\ Department of Applied Mathematics, Twente University of Technology, P.O. Box 217, $7500 \mathrm{AE}$ \\ Enschede, The Netherlands
}

\begin{abstract}
We define the concept of partial symmetry for nonlinear systems, which is an intermediate notion between the concepts of symmetry and controlled invariance. It is shown how this concept can be used for a decomposition theory of nonlinear systems and is particularly suited as a framework for treating input-output decoupling problems.
\end{abstract}

\section{Introduction}

The notion of symmetry of a dynamical system has been a subject of long standing interest in physics and mathematics. Roughly speaking, a dynamical system possesses a symmetry if its dynamics are invariant under a (coordinate) transformation or a family of (coordinate) transformations. The existence of such a symmetry implies usually that we can "decompose" the system into subsystems of lower dimension, or that we can "reduce" the system to a (quotient) system of lower dimension. In this way the knowledge of the existence of symmetries can be very useful for the qualitative understanding, or even the explicit description of the dynamics of a system. This becomes very clear in the case of Hamiltonian systems where a classical theorem of Noether asserts that symmetries are in one-to-one correspondence with the existence of conservation laws of a system (cf. $[1,2])$.

Usually the notion of symmetry is only defined for dynamical systems without inputs and outputs (for instance in the Hamiltonian case systems without external forces, cf. $[1,2])$. In recent publications $([15,16])$ the notion of symmetry is also defined for systems with inputs and outputs and a generalization of Noether's theorem is obtained. This notion of symmetry is further explored by Grizzle and Marcus ([4,3]), by using in particular families of symmetries generated by the action of a Lie group. Moreover in these papers the role of symmetries in obtaining a local or global decomposition of a system into smaller subsystems is emphasized. 
In another avenue of recent research the concept of controlled invariance for (nonlinear) systems has been used in deriving a (local) normal form of a system ([5]). The main tool in this case is the notion of a (locally) controlled invariant distribution on the state space of a system. If a system possesses such a (locally) controlled invariant distribution then, possibly after applying feedback, the system can be (locally) factored out to obtain a lower dimensional quotient system ([10]). Furthermore controlled invariant distributions and in particular the special subclass of controllability distributions have been successfully used for the input-output decoupling of a system $([8,9,11])$. Here one tries to decompose (by feedback) the system into a number of lower dimensional subsystems which are from the input-output point of view independent.

The purpose of this paper is to fill the gap between the concepts of symmetry on the one hand and controlled invariance on the other hand. In order to do so we introduce the notion of partial symmetry, which is a weaker notion than symmetry, but gives rise to a (locally) controlled invariant distribution. Since this distribution is generated by the action of a Lie group this also enables us to give a more "algebraic" treatment of controlled invariance and in particular of the input-output decoupling problems which will be considered in Section 4. In fact the concept of partial symmetry seems the natural framework for the (global) decompositions of systems aimed at in input-output decoupling.

Apart from symmetry and controlled invariance there still exists another approach to the investigation of structural properties of nonlinear systems. This approach is based on the study of the Lie algebra of vectorfields generated in a certain way by the system (the "controllability algebra"). In an innovative paper by Krener ([7]) the structure of this Lie algebra (in the case it is finite-dimensional!) is employed to derive a decomposition of the system into lower dimensional systems. The sum of the dimensions of these systems is however in general larger than the dimension of the original system. This problem is not met in the work of Respondek ([12]), where Lie algebraic conditions are stated in order to give a parallel and/or cascade decomposition of the system by using state space transformations. The controllability algebra is obviously not invariant under feedback, in contrast with the notions of symmetry and partial symmetry which we will use in this paper.

\section{Some Notation}

A coordinate free description of a general nonlinear system

$$
\dot{x}=f(x, u)
$$

can be given in the following way, see for instance [10]. Let $M$ be an $n$-dimensional state space manifold with local coordinates $x$. Let furthermore $B \stackrel{\pi}{\rightarrow} M$ be an $n+m$-dimensional fiber bundle over $M$ with projection $\pi$. We can extend the local coordinates $x$ for $M$ to local coordinates $(x, u)$ for $B$. The nonlinear system 
is now given by a smooth mapping $f: B \rightarrow T M$ such that the diagram

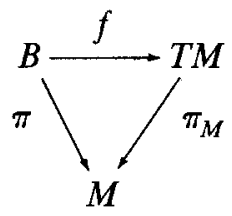

commutes.

In local coordinates $(x, u)$ this yields (1.1). A special but important case is where $B$ is a vector bundle and $f$ is an affine map. Then we obtain a representation of the form

$$
\dot{x}=A(x)+\sum_{i=1}^{m} u_{i} B_{i}(x)
$$

with $A$ and $B_{i}$ (locally) defined vectorfields on $M$. We call this an affine (or input-linear) system.

\section{Symmetries and Partial Symmetries}

In this section we define the notion of symmetry and partial symmetry for nonlinear control systems. For a more general treatment of symmetries including the inputs and the outputs of the system we refer to $[15,16]$.

The (partial) symmetries which we will deal with are generated by the action of a Lie group.

Definition 2.1 (see for instance [1]). Let $M$ be a smooth manifold. An action of a Lie group $G$ on $M$ is a smooth mapping $\Phi: G \times M \rightarrow M$ such that

(i) for all $x \in M, \Phi(e, x)=x$ ( $e$ is the identity of $G$ )

(ii) for all $x \in M, \Phi(g, \Phi(h, x))=\Phi(g h, x)$ for all $g, h \in G$ ( $g h$ is the "product" in $G$ )

We denote for each $g \in G$ the mapping $\Phi(g, \cdot): M \rightarrow M$ by $\Phi_{g}$, and the mapping $\Phi(\cdot, x): G \rightarrow M$, with $x \in M$, by $\Phi_{x}$.

We use the following additional terminology.

Definition 2.2. Let $\Phi$ be an action of $G$ on $M$. For $x \in M$ the orbit of $x$ is given by

$$
G \cdot x=\left\{\Phi_{g}(x) \mid g \in G\right\}
$$

The space of orbits is denoted by $M / G$. The projection $p_{m}: M \rightarrow M / G$ is defined by $x \rightarrow G \cdot x$. The action is free if for each $x \in M$, the map $\Phi_{x}$ is one-to-one. The action is proper if $\tilde{\Phi}: G \times M \rightarrow M \times M$, defined by $\tilde{\Phi}(g, x)=(x, \Phi(g, x))$ is a proper mapping, that is, if $K \subset M \times M$ is compact, then $\tilde{\Phi}^{-1}(K)$ is compact. 
The following statements can be proved (cf. [1]):

Proposition 2.3. Let $\Phi$ be an action of $G$ on $M$.

a. If $\Phi$ is proper, then the orbits $G \cdot x$ are closed submanifolds of $M$.

b. If $\Phi$ is free and proper, then $\Phi_{x}$ is an injective immersion.

c. If $\Phi$ is free and proper, then $M / G$ is a smooth manifold such that $p_{m}: M \rightarrow M / G$ is a surjective submersion.

After these preliminaries we give the following definition of a symmetry introduced in $[15,16]$ and followed up in $[4,3]$.

Definition 2.4. Let $\Sigma(M, B, f)$ be a nonlinear control system. Let $G$ be a Lie group such that $\Theta: G \times B \rightarrow B$ and $\Phi: G \times M \rightarrow M$ are group actions. This pair of group actions is denoted by $(G, \Theta, \Phi)$. Then $(G, \Theta, \Phi)$ is a symmetry for $\Sigma(M, B, f)$ if for each $g \in G$ the following diagram

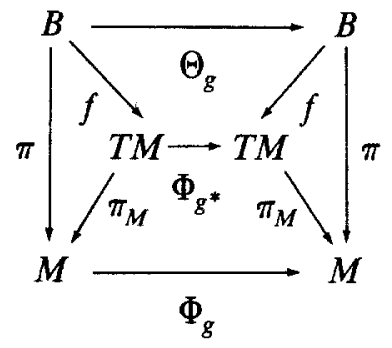

commutes, i.e.

$$
\begin{aligned}
& \pi \circ \Theta_{g}=\Phi_{g} \circ \pi \\
& f \circ \Theta_{g}=\Phi_{g^{*}} \circ f
\end{aligned} \quad \text { for all } g \in G .
$$

Example. The simplest example of a symmetry is given by a pair $(S, R)$ of complete vectorfields $S$ on $M$ and $R$ on $B$ such that the diagram

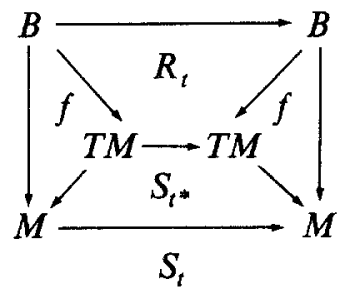

commutes for all $t \in \mathbb{R}$, with $R_{t}$ and $S_{t}$ the flows of $R$ and $S$ (i.e. $\frac{d}{d t} R_{t}(b)=$ $\left.R\left(R_{t}(b)\right), \frac{d}{d t} S_{t}(x)=S\left(S_{t}(x)\right)\right)$. In this case the group $G$ equals $\mathbb{R}$ and the group actions $\Phi: \mathbb{R} \times M \rightarrow M, \Theta: \mathbb{R} \times B \rightarrow B$ are given by $\Phi(t, x)=S_{t}(x), \Theta(t, b)=$ $R_{t}(b)$. This type of symmetry is extensively studied in $[15,16]$. 
In the sequel we will restrict ourselves to pairs of group actions $(G, \Theta, \Phi)$ such that $\Phi$ and $\Theta$ are both proper and free (Actually it is easy to prove that if $\Phi$ is proper and free, then $\Theta$ is necessarily also proper and free (cf. [4])). The orbits of $\Theta$ on $B$ define a foliation on $B$, and the orbits of $\Phi$ define a foliation on $M$ (the leaves of the foliations are the orbits of the group actions). Since $\Theta$ and $\Phi$ are free the dimension of the leaves of both foliations is equal to the dimension of $G$. Corresponding to these foliations there exist involutive distributions $D_{\Theta}$ on $B$ and $D_{\Phi}$ on $M$ (the maximal integral manifolds of $D_{\Theta}$ and $D_{\Phi}$ are the orbits of $\Theta$, respectively $\Phi)$. The dimension of both distributions is constant and equal to the dimension of $G$.

We now come to the definition of a partial symmetry. Let $f: B \rightarrow T M$ and $D$ be a distribution on $M$. Then we denote by $f(\bmod D)$ the map attaching to a point $(x, u) \in B$ the rest class $f(x, u)+D$ contained in $T M$.

Definition 2.5. Let $\Sigma(M, B, f)$ be a control system. Let $\Theta: G \times B \rightarrow B$ and $\Phi: G \times M \rightarrow M$ be group actions. Then we call $(G, \Theta, \Phi)$ a partial symmetry if for each $g \in G$

$$
\begin{aligned}
& \pi \circ \Theta_{g}=\Phi_{g} \circ \pi \\
& f \circ \Theta_{g}\left(\bmod D_{\Phi}\right)=\left(\Phi_{g}\right)_{*} \circ f\left(\bmod D_{\Phi}\right)
\end{aligned}
$$

Remark. In comparison with Definition 2.4 we have just weakened equation (2.1.b) to (2.2.b) by requiring equality modulo the distribution $D_{\Phi}$.

Let $(G, \Theta, \Phi)$ be a partial symmetry for $\Sigma(M, B, f)$. Since it will be a standing assumption that $\Theta$ and $\Phi$ are free and proper, by Proposition 2.3.c, $\tilde{B}=B / G$ and $\tilde{M}=M / G$ are smooth manifolds with submersive smooth projections $p_{b}: B \rightarrow \tilde{B}$ and $p_{m}: M \rightarrow \tilde{M}$. Moreover the following holds

Proposition 2.6. $\pi: B \rightarrow M$ induces a smooth mapping $\tilde{\pi}: \tilde{B} \rightarrow \tilde{M}$ such that

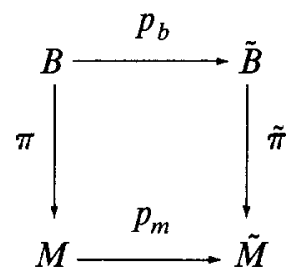

commutes, and $\tilde{B} \stackrel{\tilde{\pi}}{\rightarrow} \tilde{M}$ is a fiber bundle with standard fiber equal to the standard fiber of $B$. In fact $B \stackrel{M}{\rightarrow} M$ is diffeomorphic to the pullback bundle (over $p_{m}$ ) of $\tilde{B} \stackrel{\tilde{\pi}}{\rightarrow} \tilde{M}$.

Proof. Since $\pi$ is by $(2.2 . a)$ equivariant $([1$, p. 264]) with respect to the actions $\Phi$ and $\Theta, \pi$ projects to a smooth mapping $\tilde{\pi}: \tilde{B} \rightarrow \tilde{M}$ ("passage to quotients"). Since $\pi$ is a surjective submersion it follows that $\tilde{\pi}$ is a surjective submersion too. 
From the definition of the differentiable structure on $\tilde{M}$ and $\tilde{B}$ it follows that $\tilde{B} \stackrel{\tilde{\pi}}{\rightarrow} \tilde{M}$ is a fiber bundle with standard fiber equal to the standard fiber of $B \stackrel{\pi}{\rightarrow} M\left(\right.$ since $\left.\operatorname{dim} D_{\Theta}=\operatorname{dim} D_{\Phi}\right)$.

Remark. Therefore the condition (2.2.b) for a partial symmetry

$$
f \circ \Theta_{g}\left(\bmod D_{\Phi}\right)=\left(\Phi_{g}\right)_{*} \circ f\left(\bmod D_{\Phi}\right)
$$

can be also stated as

$$
\left(p_{m}\right)_{*} \circ f \circ \Theta_{g}=\left(p_{m}\right)_{*} \circ\left(\Phi_{g}\right)_{*} \circ f
$$

Actually not only the fiber bundle $B \stackrel{\pi}{\rightarrow} M$ projects a fiber bundle $\tilde{B} \stackrel{\tilde{m}}{\rightarrow} \tilde{M}$ but the whole control system projects to a control system $\tilde{\Sigma}(\tilde{M}, \tilde{B}, \tilde{f})$ :

Proposition 2.7. Let $(G, \Theta, \Phi)$ be a partial symmetry for a nonlinear control system $\Sigma(M, B, f)$ (with $\Theta$ and $\Phi$ free and proper). Then there exists a smooth mapping $\tilde{f}: \tilde{B} \rightarrow T \tilde{M}$ such that the diagram

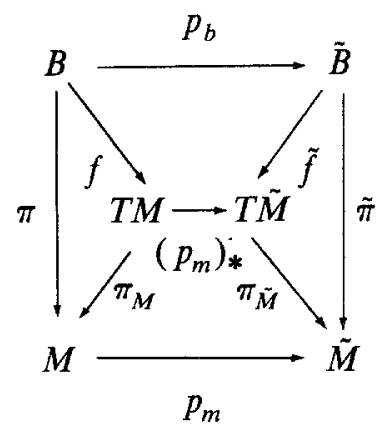

commutes.

Proof. Define $\tilde{f}$ by $\tilde{f}(\tilde{b}):=\left(p_{m}\right)_{*} f(b)$, with $b \in p_{b}^{-1}(\tilde{b})$. It follows from (2.2.b) that $\tilde{f}$ is a well-defined smooth mapping which makes the diagram commutative.

Remark 1. The control system $\tilde{\Sigma}(\tilde{M}, \tilde{B}, \tilde{f})$ as above is called a quotient system (see [10]).

Remark 2. Of course, Propositions 2.6 and 2.7 hold a fortiori for symmetries $(G, \Theta, \Phi)$ (Definition 2.4.)

We now investigate what the existence of a symmetry or partial symmetry implies for the structure of the control system. First we make a local analysis. Let $(G, \Theta, \Phi)$ be a (partial) symmetry, and let $D_{\Theta}$ and $D_{\Phi}$ be the corresponding distributions on $B$ and $M$. 
Since $D_{\Phi}$ is integrable we can locally choose coordinates $x=\left(x_{1}, \ldots, x_{n}\right)$ for $M$ such that $D_{\Phi}=\operatorname{span}\left\{\frac{\partial}{\partial x_{1}}, \ldots, \frac{\partial}{\partial x_{k}}\right\}$, with $k \leq n$ (Frobenius). Furthermore we can consider the coordinates $\left(x_{1}, \ldots, x_{n}\right)$ for $M$ as functions on $B$ (by identifying them with $\left.x_{i} \circ \pi\right)$, and extend this set of functions to coordinates $(x, u)=$ $\left(x_{1}, \ldots, x_{n}, u_{1}, \ldots, u_{m}\right)$ for $B$. We call such coordinates for $B$ fiber respecting. Moreover since $D_{\Theta}$ is integrable and $\pi_{*} D_{\Theta}=D_{\Phi}$ (this follows from $\pi \circ \Theta_{g}=$ $\Phi g \circ \pi, \forall g$ ), we can choose the coordinate functions $u_{1}, \ldots, u_{m}$ in such a way that with respect to the coordinates $(x, u)\left(\right.$ remember $\left.\operatorname{dim} D_{\Theta}=\operatorname{dim} D_{\Phi}\right)$

$$
D_{\Theta}=\operatorname{span}\left\{\frac{\partial}{\partial x_{1}}, \ldots, \frac{\partial}{\partial x_{k}}\right\} \quad(\operatorname{see}[10])
$$

Denote for simplicity $x^{1}=\left(x_{1}, \ldots, x_{k}\right)$ and $x^{2}=\left(x_{k+1}, \ldots, x_{n}\right)$. Then, if $(G, \Theta, \Phi)$ is a partial symmetry, it follows from $f \circ \Theta_{g}\left(\bmod D_{\Phi}\right)=\left(\Phi_{g}\right)_{*} \circ f\left(\bmod D_{\Phi}\right), \forall g \in$ $G$, that the system has the following normal form

$$
\begin{aligned}
& \dot{x}^{1}=f^{1}\left(x^{1}, x^{2}, u\right) \\
& \dot{x}^{2}=f^{2}\left(x^{2}, u\right)
\end{aligned}
$$

with $f=\left(f^{1}, f^{2}\right)$ in the above coordinates $\left(x^{1}, x^{2}\right)$.

A fortiori this normal form holds if $(G, \Theta, \Phi)$ is a symmetry. However in this case we can say more following [3, 4]. Let the center (the maximal abelian sub group) of $G$ be $l$-dimensional $(l \leq k)$. Then it is easily seen that we can choose the coordinates $\left(x_{1}, \ldots, x_{k}\right)$ in such a way that the vectorfields $\frac{\partial}{\partial x_{1}}, \ldots, \frac{\partial}{\partial x_{l}}$ are the images of left-invariant vectorfields on $G$ under the action $\Phi$, i.e. there exist independent vectors $\xi_{1}, \ldots, \xi_{l}$ in $T_{e} G$ such that for every $x$ in the coordinate neighbourhood

$$
\frac{\partial}{\partial x_{i}}=\left.\frac{d}{d t} \Phi\left(\exp t \xi_{i}, x\right)\right|_{t=0}, \quad i=1, \ldots, l .
$$

where exp: $T_{e} G \rightarrow G$ is the usual exponential map. Then, if $(G, \Theta, \Phi)$ is a symmetry, $f \circ \Theta_{g}=\left(\Phi_{g}\right)_{*} \circ f$, for each $g \in G$, implies that the system has the following more specialized normal form

$$
\begin{array}{lcc}
\dot{x}_{1} & =f_{1} & \left(x_{l+1}, \ldots, x_{n}, u\right) \\
\vdots & \vdots & \\
\dot{x}_{l}=f_{l} & \left(x_{l+1}, \ldots, x_{n}, u\right) \\
\dot{x}_{l+1} & =f_{l+1} & \left(x_{l+1}, \ldots, x_{n}, u\right) \\
\vdots & \vdots & \\
\dot{x}_{k}=f_{k} & \left(x_{l+1}, \ldots, x_{n}, u\right) \\
\dot{x}_{k+1}=f_{k+1} & \left(x_{k+1}, \ldots, x_{n}, u\right) \\
\vdots & \vdots & \\
\dot{x}_{n}=f_{n} & \left(x_{k+1}, \ldots, x_{n}, u\right)
\end{array}
$$


In particular if $G$ is abelian $(l=k)$ we can choose the coordinates $x^{1}=\left(x_{1}, \ldots, x_{k}\right)$ in such a way that the system has the form

$$
\begin{aligned}
& \dot{x}^{1}=f^{1}\left(x^{2}, u\right) \\
& \dot{x}^{2}=f^{2}\left(x^{2}, u\right)
\end{aligned}
$$

For a global analysis we make use of some results obtained in [4]. We define feedback as a bundle isomorphism from $B$ to itself (cf. $[9,15])$. So feedback is a diffeomorphism $\alpha: B \rightarrow B$ such that the diagram $B \underset{\pi}{\longrightarrow} B$ commutes.

We call a control system $\Sigma(M, B, f)$ feedback equivalent to another control system $\Sigma^{\prime}\left(M, B, f^{\prime}\right)$ if there exists a feedback $\alpha$ such that $f^{\prime}=f \circ \alpha$ (cf. $\left.[10,16]\right)$. The following proposition is immediate (cf. [4]).

Proposition 2.8. Suppose $\Sigma(M, B, f)$ has a (partial) symmetry $(G, \Theta, \Phi)$. Let $\Psi$ be another G-action on B satisfying $\pi \circ \Psi_{g}=\Phi_{g} \circ \pi, \forall g \in G$. Then $\Sigma$ is feedback equivalent to some system having a (partial) symmetry $(G, \Psi, \Phi)$ if and only if there exists a feedback $\alpha: B \rightarrow B$ satisfying $f \circ \Theta_{g} \circ \alpha=f \circ \alpha \circ \Psi_{g}, \forall g \in G$, in the case of a symmetry, or, $f \circ \Theta_{g} \circ \alpha\left(\bmod D_{\Phi}\right)=f \circ \alpha \circ \Psi_{g}\left(\bmod D_{\Phi}\right), \forall g \in G$, in the case of a partial symmetry.

A sufficient condition for the existence of a feedback $\alpha$ is stated in the following

Proposition 2.9. Take the same assumptions as in Proposition 2.8. Furthermore suppose that $M$ is diffeomorphic to $M / G \times G$. Then there exists a feedback $\alpha$ satisfying $\Theta_{g} \circ \alpha=\alpha \circ \Psi_{g}, \forall g \in G$.

Remark. In [4] this proposition is proved for trivial bundles $B=M \times U$, but the proof is easily generalized to arbitrary fiber bundles.

For the global analysis of the structure induced by the existence of a symmetry or a partial symmetry we will now make the following rather severe assumptions following [4]:

1. $B$ is trivial, i.e. $B=M \times U$, with $U$ a manifold,

2. $M$ is diffeomorphic to $M / G \times G$.

Let now $(G, \Theta, \Phi)$ be a (partial) symmetry for the control system $\Sigma(M, B, f)$. Consider the $G$-action $\Psi$ on $B(=M \times U)$ defined by

$$
\Psi_{g}(m, u)=\left(\Phi_{g}(m), u\right) \quad m \in M, u \in U
$$

It is clear that $\pi \circ \Psi_{g}=\Phi_{g} \circ \pi$. Hence by propositions $2.8,2.9$ there exists a feedback $\alpha$ such that $\Sigma(M, B, f)$ is feedback equivalent to the control system $\Sigma^{\prime}\left(M, B, f^{\prime}\right)$, with $f^{\prime}=f \circ \alpha$, having (partial) symmetry $(G, \Psi, \Phi)$. Since $M \simeq$ $M / G \times G$ we can identify a point $m \in M$ with a point $(y, g) \in M / G \times G$. Then 
if $(G, \Theta, \Phi)$ is a symmetry we obtain the following global normal form for the system $\Sigma^{\prime}\left(M, B, f^{\prime}\right)$. The system equations $\dot{x}=f^{\prime}(x, u)$ take the form (see [4])

$$
\begin{aligned}
& \dot{y}=\tilde{f}^{\prime}(y, u) \\
& \dot{g}=T_{e} \mathscr{L}_{g} v,
\end{aligned}
$$

with $v=h(y, u)$ and $\mathscr{L}_{g}$ the left multiplication on $G$. Here $\tilde{f}^{\prime}$ is the "quotient mapping" of $f^{\prime}$ (see Proposition 2.7), while the mapping $h$ is further specified in [4]. We notice that both mappings $\tilde{f}^{\prime}$ and $h$ do not depend on $g$.

Similarly, if $(G, \Theta, \Phi)$ is only a partial symmetry we obtain the following normal form for $\dot{x}=f^{\prime}(x, u)$

$$
\begin{aligned}
& \dot{y}=\tilde{f}^{\prime}(y, u) \\
& \dot{g}=h(y, g, u)
\end{aligned}
$$

with $h$ a certain mapping generally depending on $g$. Schematically we summarize the situation as follows (cf. [3, 4]).

Symmetry. $\Sigma\left(M, B, f^{\prime}\right)$ has the form

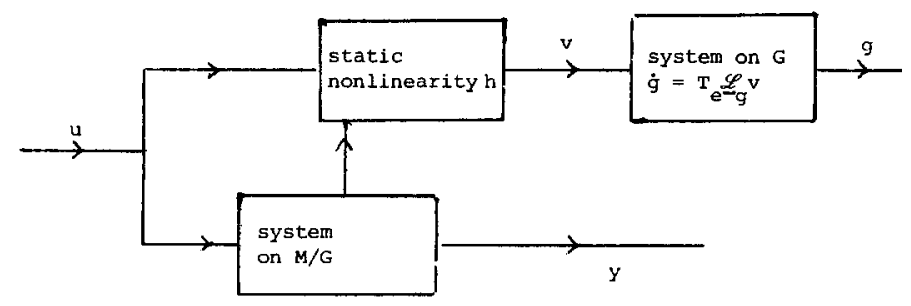

Partial Symmetry. $\Sigma\left(M, B, f^{\prime}\right)$ has the form

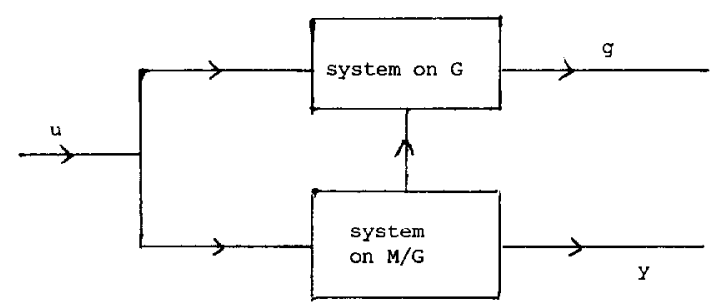

By taking the feedback $\alpha$ into account one easily obtains similar decompositions of the original system $\Sigma(M, B, f)$.

\section{Connections with Controlled Invariance}

Let $(G, \Theta, \Phi)$ be a partial symmetry for a nonlinear control system $\Sigma(M, B, f)$ and let the distributions $D_{\Theta}$ and $D_{\Phi}$ be as before. Recall the definition of the 
prolongation $\dot{D}$ of an involutive distribution $D$ of constant dimension on $M$ (see [10]). In local coordinates $\dot{D}$ is given as follows. By Frobenius' Theorem we can choose coordinates $\left(x_{1}, \ldots, x_{n}\right)$ for $M$ such that $D=\operatorname{span}\left\{\frac{\partial}{\partial x_{1}}, \ldots, \frac{\partial}{\partial x_{k}}\right\}, k \leq n$. Then $\dot{D}$ is the distribution on $T M$ locally given by

$$
\dot{D}=\operatorname{span}\left\{\frac{\partial}{\partial x_{1}}, \ldots, \frac{\partial}{\partial x_{k}}, \frac{\partial}{\partial \dot{x}_{1}}, \ldots, \frac{\partial}{\partial \dot{x}_{k}}\right\}
$$

Here $\left(x_{1}, \ldots, x_{n}, \dot{x}_{1}, \ldots, \dot{x}_{n}\right)$ denote coordinates for $T M$ with $\dot{x}_{i}: T M \rightarrow \mathbb{R}$ defined by $\dot{x}_{i}(v)=d x_{i}(v)$ for $v \in T M$.

Recall furthermore that an involutive distribution $D$ of constant dimension on $M$ is called locally controlled invariant w.r.t. the control system $\Sigma(M, B, f)$ if locally around each point on $M$ there exists an involutive distribution $E$ of constant dimension on $B$ such that (see [10])

1. $\pi_{*} E=D$ and $\operatorname{dim} E=\operatorname{dim} D$

2. $f_{*} E \subset \dot{D}$

We obtain

Proposition 3.1. Let $(G, \Theta, \Phi)$ be a partial symmetry for $\Sigma(M, B, f)$. Then: $\pi_{*} D_{\Theta}=D_{\Phi}$, $\operatorname{dim} D_{\Theta}=\operatorname{dim} D_{\Phi}$ and $f_{*} D_{\Theta} \subset \dot{D}_{\Phi}$. Hence $D_{\Phi}$ is locally controlled invariant.

Proof. $\pi_{*} D_{\Theta}=\dot{D_{\Phi}}$ follows from (2.2.a), while $f_{*} D_{\Theta} \subset \dot{D}_{\Phi}$ follows from differentiating (2.2.b) along curves $t \mapsto g(t)$ in the Lie group $G$.

We conclude that a partial symmetry, and a fortiori a symmetry, yields a locally controlled invariant distribution $D_{\Phi}$. This distribution however has a richer structure than a general locally controlled invariant distribution $D$, because of the following reasons:

1. The distribution $E$ on $B$ such that $\operatorname{dim} E=\operatorname{dim} D, \pi_{*} E=D, f_{*} E \subset \dot{D}$ is globally defined if $D=D_{\Phi}$, with $(G, \Theta, \Phi)$ a partial symmetry. Indeed $E=D_{\Theta}$.

2. The distributions $D_{\Phi}$ and $D_{\Theta}$ have a special structure, being generated by a group action. In fact $D$, viewed as a subbundle of $T M$, is a trivial bundle over $M$, isomorphic to $M \times g(g$ is the Lie algebra of $G)$. The same applies to $D_{\Theta}$.

3. If $\Phi$ and $\Theta$ are free and proper then $M / G$ and $B / G$ are well-defined smooth quotient manifolds.

We recall from [10] that a necessary and sufficient condition for an involutive distribution $D$ of constant dimension on $M$ to be locally controlled invariant is that $D$ satisfies

$$
f_{*}\left(\pi_{*}^{-1}(D)\right) \subset \dot{D}+f_{*}\left(\Delta_{0}^{e}\right)
$$

where $\Delta_{0}^{e} \subset T B$ is the vertical tangent space of $B$, i.e. $\Delta_{0}^{e}(b)=\left\{X \in T_{b} B \mid \pi_{*} X=\right.$ $0\}, \forall b \in B$. In fact this condition implies the local existence of a distribution $E$ 
on $B$ satisfying (3.1). If we assume that $D$ satisfies (3.2) and is generated by a group action we can say more:

Proposition 2.1. Let $\Phi: G \times M \rightarrow M$ be a group action (free and proper). Let $D_{\Phi}$ be locally controlled invariant (i.e. satisfies (3.2)). Assume that $G$ is simply connected. Then there exists a globally defined distribution $E$ on $B$ such that

$$
\begin{aligned}
& f_{*} E \subset \dot{D}_{\Phi} \\
& \pi_{*} E=D \text { and } \operatorname{dim} E=\operatorname{dim} D_{\Phi}
\end{aligned}
$$

Furthermore assume that $E$ defines a horizontally complete connection (cf. [10]) in the bundle $B$ above every leaf of the foliation corresponding to $\Phi$. Then there exists $a$ group action $\Theta: G \times B \rightarrow B$ (free and proper) such that $E=D_{\Theta}$. Hence $(G, \Theta, \Phi)$ is a partial symmetry.

Proof. $M / G$ is a smooth manifold such that $p_{m}: M \rightarrow M / G$ is a smooth submersion. Because $D_{\Phi}$ is locally controlled invariant $E$ satisfying (3.3) exists locally. Since $G$ is simply connected the leaves of the foliation of $M$ induced by $D_{\Phi}$ are also simply connected. Then it follows (see [6]) that $E$ exists globally. Finally we construct $\Theta: G \times B \rightarrow B$ as follows. Let $g \in G$ and $b \in B$. Denote the maximal integral manifold of $E$ through $B$ by $F$. Now there exists a unique point $b^{\prime} \in F$ such that $\pi\left(b^{\prime}\right)=\Phi_{g}(\pi(b))$. (The existence follows from the fact that $E$ defines a horizontally complete connection, and so we can lift every curve in a leaf of $D_{\Phi}$ to a curve in $B$. The uniqueness follows from $G$ being simply connected). Then define $\Theta_{g}(b)=b^{\prime}$. It is easy to see that $\Theta_{g}$ is a smooth group action.

Remark. In the next section we will restrict ourselves to affine (or input-linear) systems $\dot{x}=A(x)+B(x) u$. In this case the distribution $E$ is an affine connection on $B$ (above every leaf) given by tangent vectors of the form

$$
\frac{\partial}{\partial x_{i}}+\left[h_{i}(x)+K_{i}(x) u\right] \frac{\partial}{\partial u}
$$

where the $h_{i}$ and $K_{i}$ satisfy certain integrability conditions (cf. [10]). Such affine connections are automatically horizontally complete. So in this case we do not have to make extra assumptions in order to define $\Theta$ such that $E=D_{\Theta}$.

The contents of the last proposition are related to the notion of global controlled invariance. Recall $([10,16])$ that we call a distribution $D$ on $M$ globally controlled invariant if there exists a horizontal involutive distribution $H$ on $B$ (horizontal means $\operatorname{dim} H=\operatorname{dim} M$ and $\pi_{*} H=T M$, i.e. an integrable connection cf. [10]), such that

$$
f_{*}\left(\pi_{*}^{-1}(D) \cap H\right) \subset \dot{D}
$$


We derive

Proposition 3.3. Let $(G, \Theta, \Phi)$ be a partial symmetry. Suppose that the bundle $\tilde{B}=B / G$ admits a horizontal involutive distribution $\tilde{H}$. Then $D_{\Phi}$ is globally controlled invariant.

Proof. There exists a unique horizontal involutive distribution $H$ on $B$ such that $\left(p_{b}\right)_{*} H=\tilde{H}$ and $\operatorname{ker}\left(p_{b}\right)_{*} \cap H=D_{\Theta}$. This distribution $H$ does the job.

\section{Decomposition and Partial Symmetries}

In this section we will restrict ourselves to smooth affine nonlinear systems $\Sigma(M, B, f)$ :

$$
\dot{x}=A(x)+\sum_{i=1}^{m} B_{i}(x) u_{i}=A(x)+B(x) u
$$

It is assumed that the distribution $\Delta_{0}:=\operatorname{Span}\left\{B_{1}, \ldots, B_{m}\right\}$ has constant dimension $m$ on $M$. Our goal is to discuss here the relation between partial symmetries and decomposition of nonlinear systems. In our context decomposition can be understood in three different ways:

1. algebraically: The group action $(G, \Theta, \Phi)$ has a refined algebraic structure.

2. geometrically: The distributions $D_{\Theta}$ and $D_{\Phi}$ induced by a partial symmetry possess a special geometric structure.

3. analytically: The normal form (2.3) associated with a partial symmetry $(G, \Theta, \Phi)$ has a more detailed structure.

The second characterization enables us to fully exploit the recent results on input-output decoupling problems of a nonlinear system, see $[8,11,12,13]$. It is our belief that the notion of partial symmetry is a natural framework for the (global) study of these noninteracting control problems.

To start with, we assume there is given a Lie-group $G$ together with closed Lie-subgroups $G_{1}, \ldots, G_{k}$. Let $\Phi$ be a free and proper action of $G$ on $M$. Then $\Phi$ induces free and proper actions $\Phi_{i}$ of $G_{i}$ on $M$ by

$$
\Phi_{i}=\left.\Phi\right|_{G_{i}}, \quad i=1, \ldots, k \text {. }
$$

Suppose that there exist partial symmetries $\left(G_{1}, \Theta_{1}, \Phi_{1}\right), \ldots,\left(G_{k}, \Theta_{k}, \Phi_{k}\right)$ for the affine system $\Sigma(M, B, f)$ given by (4.1). Our first results treats the simplest case, where the subgroups $G_{1}, \ldots, G_{k}$ are 'independent', i.e.

$$
G=G_{1} \oplus \cdots \oplus G_{k}
$$

Lemma 4.1. Let $G, G_{1}, \ldots, G_{k}$ be Lie-groups satisfying (4.3), with proper and free group actions $\Phi, \Phi_{1}, \ldots, \Phi_{k}$ satisfying (4.2). Let $\left(G_{1}, \Theta_{1}, \Phi_{1}\right), \ldots,\left(G_{k}, \Theta_{k}, \Phi_{k}\right)$ be partial symmetries for the strongly accessible (cf. [14]) system (4.1). Then there 
exists a partial symmetry $(G, \Theta, \Phi)$ for (4.1) if

$$
\Delta_{0}=\sum_{i \in \underline{k}} \Delta_{0} \cap D_{\Phi_{i}}
$$

Proof. By the fact that $\Phi: G \times M \rightarrow M$ is a free action and while (4.3) holds, it follows that the induced involutive distributions $D_{\Phi_{1}}, \ldots, D_{\Phi_{k}}$ are independent, cf. $[13,8]$, i.e. the intersection of distinct unions of $D_{\Phi_{i}}$ 's is zero $\left(\sum_{i \in I} D_{\Phi_{i}} \cap \sum_{j \in J} D_{\Phi_{j}}=0\right.$ for $I, J \subset \underline{k}, I \cap J=\varnothing)$. Notice that $\operatorname{dim}\left(D_{\Phi_{i}}\right)=\operatorname{dim} G_{i}, i \in k$. From the strong accessibility of (4.1) and the fact that $\Delta_{0}=\sum_{i \in \underline{k}} \Delta_{0} \cap D_{\Phi_{i}} \subset$
$D_{\Phi}$ it follows, see [16],

$$
\operatorname{dim}\left(D_{\Phi}\right)=\operatorname{dim}(T M)=n,
$$

with $T M$ the maximal distribution on $M$. Furthermore the distributions $D_{\Phi_{1}}, \ldots, D_{\Phi_{k}}$ are simultaneously integrable, see [13], therefore

$$
T M=D_{\Phi}=D_{\Phi_{1}} \oplus \cdots \oplus D_{\Phi_{k}},
$$

cf. $[8,13]$.

In order to prove that there exists a partial symmetry $(G, \Theta, \Phi)$ for (4.1) we only need to construct the free and proper action $\Theta: G \times B \rightarrow B$. But the so-called noninteracting condition (4.4) guarantees that the distributions $D_{\Phi_{1}}, \ldots, D_{\Phi_{k}}$ (all being locally controlled invariant, cf. section 3 ) are compatible, i.e. there locally exists a state feedback for the system (4.1) such that each of the distributions $D_{\Phi_{1}}, \ldots, D_{\Phi_{k}}$ is invariant for the modified system (remark (iii), p. 17 of [11]). From this and proposition 3.2 (also remark after proposition 3.2) it now follows that there is a free and proper group action $\Phi: G \times B \rightarrow B$.

Remark. It is emphasized that in general $\Theta \mid G_{i} \neq \Theta_{i}$.

Corollary 4.2. Under the conditions of lemma 4.1 the normal form (2.3) associated with the partial symmetry $(G, \Theta, \Phi)$ has the following detailed structure:

$$
\begin{aligned}
& \dot{x}^{1}=\tilde{A}^{1}\left(x^{1}\right)+\tilde{B}^{1}\left(x^{1}\right) u^{1} \\
& \dot{x}^{i}=\tilde{A}^{i}\left(x^{i}\right)+\tilde{B}^{i}\left(x^{i}\right) u^{i} \\
& \dot{x}^{k}=\tilde{A}^{k}\left(x^{k}\right)+\tilde{B}^{k}\left(x^{k}\right) u^{k}
\end{aligned}
$$

Proof. Because the independent distributions $D_{\Phi_{1}}, \ldots, D_{\Phi_{k}}$ are simultaneously integrable we may choose local coordinates $x=\left(x_{1}, \ldots, x_{n}\right)=\left(x^{1}, \ldots, x^{k}\right)$ such that, cf. [13],

$$
D_{\Phi_{i}}=\operatorname{span}\left\{\frac{\partial}{\partial x^{i}}\right\}, \quad i \in \underline{k} .
$$

The result now follows from [8]. 
Our second result in this context deals with the situation that the closed Lie-subgroups $G_{1}, \ldots, G_{k}$ are no longer independent. Perhaps somewhat surprisingly condition (4.3) may be replaced by the much weaker version ( $U$ is the set-theoretic union)

$$
G=G_{1} \cup \cdots \cup G_{k} .
$$

Obviously we still insist on the constraint that the free and proper actions $\Phi_{i}$ of $G_{i}$ on $M$ are induced by the action $\Phi$, i.e.

$$
\Phi_{i}=\left.\Phi\right|_{G_{i}}, \quad i \in \underline{k} .
$$

Notice that in contrast with (4.2) and (4.3), the constraints (4.8) and (4.9) have certain implications for the actions $\Phi_{i}, i \in \underline{k}$. For example $\Phi_{i}$ and $\Phi_{j}$ coincide on the subgroup $G_{i} \cap G_{j}, i, j \in \underline{k}$.

Lemma 4.3. Let $G$ be a Lie group with closed Lie subgroups $G_{1}, \ldots, G_{k}$, satisfying (4.8), with proper and free actions $\Phi, \Phi_{1}, \ldots, \Phi_{k}$ satisfying (4.9). Let $\left(G_{1}, \Theta_{1}, \Phi_{1}\right), \ldots,\left(G_{k}, \Theta_{k}, \Phi_{k}\right)$ be partial symmetries for the strongly accessible system (4.1). Then there exists a partial symmetry $(G, \Theta, \Phi)$ for the control system (4.1) if

$$
\Delta_{0}=\sum_{i \in \underline{k}} \Delta_{0} \cap D_{\Phi_{i}}
$$

Proof. This result follows the same lines as lemma 4.1. The basic observation is that (4.10) is the necessary and sufficient condition for compatibility of the distributions $D_{\Phi_{1}}, \ldots, D_{\Phi_{k}}$, see $[11,12]$. In the same way as in lemma 4.1 we may conclude that there exists a free and proper action $\Phi: G \times B \rightarrow B$.

Remark. As in Lemma 4.1 the involutivity of the distribution $\sum_{i \in I} D_{\Phi_{i}}, I \subset \underline{k}$, is a direct consequence of the group action $\left.\Phi\right|_{\sum_{i \in I} G_{i}}: \sum_{i \in I} G_{i} \times M \rightarrow M$. In the geometric treatment of input-output decoupling problems this integrability issue is hard to solve, see $[11,12]$.

Corollary 4.4. Under the conditions of lemma 4.3 the normal form associated with the partial symmetry $(G, \Theta, \Phi)$ has the following detailed structure

$$
\begin{array}{ll}
\dot{x}^{1} & =\tilde{A}^{1}\left(x^{1}\right)+\tilde{B}^{1}\left(x^{1}\right) u^{1} \\
\vdots & \vdots \\
\dot{x}^{i} & =\tilde{A}^{i}\left(x^{i}\right)+\tilde{B}^{i}\left(x^{i}\right) u^{i} \\
\vdots & \vdots \\
\dot{x}^{k} & =\tilde{A}^{k}\left(x^{k}\right)+\tilde{B}^{k}\left(x^{k}\right) u^{k} \\
\dot{x}^{k+1} & =\tilde{A}\left(x^{1}, \ldots, x^{k+1}\right)+\sum_{i \in \underline{m}} \tilde{B}_{i}\left(x^{1}, \ldots, x^{k+1}\right) u_{i}
\end{array}
$$

Proof. See [11, revised version]. 
Up till now we assumed that a group action of $G$ on $M$ was already given. A more general question deals with the problem of first defining an action of $G$ on $M$, given the actions of $G_{i}$ on $M$ and afterwards constructing the action of $G$ on $B$. The following result should be considered as the preliminaries for lemmas 4.1 and 4.3.

Lemma 4.5. Suppose $\left(G_{1}, \Theta_{1}, \Phi_{1}\right), \ldots,\left(G_{k}, \Theta_{k}, \Phi_{k}\right)$ are partial symmetries for the strongly accessible affine system (4.1). Suppose that the distributions $D_{\Phi_{1}}, \ldots, D_{\Phi_{k}}$ are independent and simultaneously integrable. Then there exists a group action $\Phi: G \times M \rightarrow M$ with $G=G_{1} \oplus \cdots \oplus G_{k}$ and $\left.\Phi\right|_{G_{i}}=\Phi_{i}, i \in \underline{k}$.

Proof. From the fact that the distributions $D_{\Phi_{1}}, \ldots, D_{\Phi_{k}}$ are simultaneously integrable it follows that for $g_{i} \in G_{i}$ the map $\Phi_{i}\left(g_{i}, \cdot\right): M \rightarrow M$ leaves the distribution $D_{\Phi_{j}}$ invariant, $i, j \in \underline{k}$.

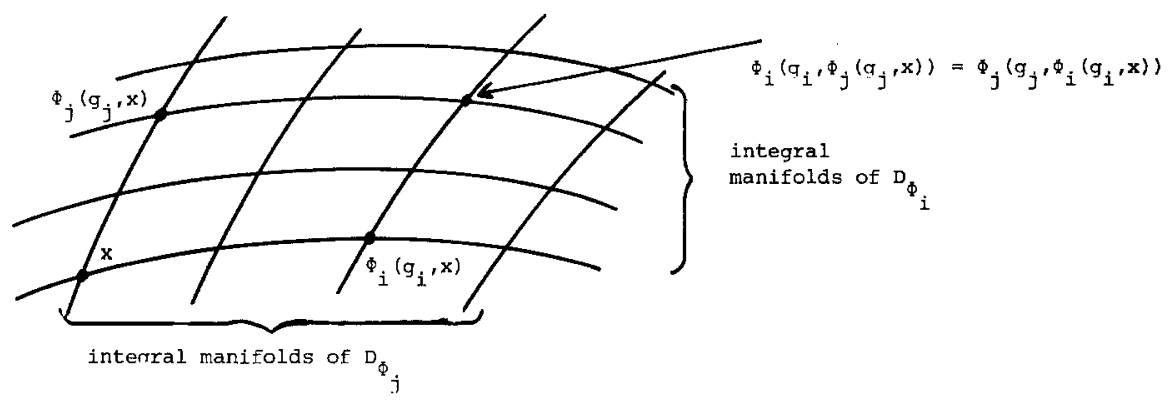

Namely, while $D_{\Phi_{i}}+D_{\Phi_{j}}$ is involutive, we have that there is a well defined distribution $D_{\Phi_{i}}$ on $M / G_{j}=M / D_{\Phi_{j}}$ such that $p_{j *}\left(D_{\Phi_{i}}\right)=\tilde{D}_{\Phi_{i}}$, where $p_{j}: M \rightarrow$ $M / D_{\Phi_{j}}$ is the corresponding projection. Notice that the integral manifolds of $\tilde{D}_{\Phi_{i}}$ are diffeomorphic to the integral manifolds of $D_{\Phi_{i}}\left(D_{\Phi_{i}}\right.$ and $D_{\Phi_{j}}$ are independent). Therefore there exists a well defined Lie-group action $\tilde{\Phi}_{j}: G_{j} \times M / D \Phi_{i} \rightarrow M / D \Phi_{i}$ by

$$
\tilde{\Phi}_{j}\left(g_{j}, x \bmod D_{\Phi_{i}}\right)=\Phi_{j}\left(g_{j}, x\right) \bmod D_{\Phi_{i}} .
$$

Notice that the above procedure also works for more than two distributions $D_{\Phi_{i}}$ and $D_{\Phi_{i}}$.

Next we come to the definition of $\Phi: G \times M \rightarrow M$, where $G=G_{1} \oplus \cdots \oplus G_{k}$. Each $g \in G$ has a unique representation as

$$
g=g_{1}+\cdots+g_{k}, \quad \text { where } g_{i} \in G_{i}, i \in \underline{k} .
$$

Define

$$
\Phi(g, \cdot)=\Phi_{1}\left(g_{1}, \Phi_{2}\left(g_{2}, \ldots, \Phi_{k-1}\left(g_{k-1}, \Phi_{k}\left(g_{k}, \cdot\right)\right)\right)\right) .
$$

It is now easy to verify that $\Phi$ is a group action of $G$ on $M$, which is again proper 
and free (the actions $\Phi_{i}$ of $G_{i}$ on $M$ are proper and free) and moreover satisfy

$$
\left.\Phi\right|_{G_{i}}=\Phi_{i}, \quad i \in \underline{k} .
$$

Also, because $\Phi_{i}\left(g_{i}, \cdot\right)$ leaves the distribution $D_{\Phi_{i}}$ invariant, we may replace the order of terms in the right-hand side of (4.12) in an arbitrary way.

Putting together lemma 4.1 and lemma 4.5 we arrive at

Corollary 4.6. Suppose $\left(G_{1}, \Theta_{1}, \Phi_{1}\right), \ldots,\left(G_{k}, \Theta_{k}, \Phi_{k}\right)$ are partial symmetries for the strongly accessible system (4.1). Suppose that the distributions are independent and simultaneously integrable. Then there exists a partial symmetry $(G, \Theta, \Phi)$ for (4.1), with $G=G_{1} \oplus \cdots \oplus G_{k}$ and $\left.\Phi\right|_{G_{i}}=\Phi_{i}, i \in \underline{k}$, if

$$
\Delta_{0}=\sum_{i \in \underline{k}} \Delta_{0} \cap D_{\Phi_{i}}
$$

Remark. We note that in fact the strong accessibility assumption in the foregoing lemmas is superfluous. This is most easily seen by reducing the state space by factoring out the 'uncontrollable part'.

Next we generalize the foregoing for the case that the Lie-groups $G_{1}, \ldots, G_{k}$ are no longer independent. So we will start with a Lie-group $G$ with closed Lie-subgroups $G_{1}, \ldots, G_{k}$. As we have seen in lemma 4.3 the simultaneous integrability of the distributions $D_{\Phi_{1}}, \ldots, D_{\Phi_{k}}\left(\Phi_{i}: G_{i} \times M \rightarrow M\right.$ group actions), i.e., for all $i \in \underline{k}$ is $\sum_{i \in I} D_{\Phi_{i}}$ involutive, plays a crucial role. Independency of the $G_{i}$ 's is not a sufficient condition for simultaneous integrability as can be seen from the following example.

Example 4.7. Let $G=S O(3)$ and its Lie algebra $g=s o(3)$. Define the Lie subalgebras $g_{1}$ and $g_{2}$ as

$$
g_{1}=\operatorname{span}\left[\begin{array}{rrr}
0 & 1 & 0 \\
-1 & 0 & 0 \\
0 & 0 & 0
\end{array}\right], \quad g_{2}=\operatorname{span}\left[\begin{array}{rrr}
0 & 0 & 1 \\
0 & 0 & 0 \\
-1 & 0 & 0
\end{array}\right]
$$

Let $G_{1}$ and $G_{2}$ be the corresponding Lie subgroups, $G_{1}=\exp g_{1}, G_{2}=\exp g_{2}$. Although $g_{1}$ and $g_{2}$ are independent the set-theoretic union of $g_{1}$ and $g_{2}$, $g_{1} \cup g_{2}$, is not a Lie subalgebra of $g$ and therefore also $G_{1} \cup G_{2}$ is not a Lie-subgroup of $G$.

In the following easy proposition the necessary and sufficient conditions for simultaneous integrability are given.

Proposition 4.8. Let $G$ be a Lie group with closed subgroups $G_{1}, \ldots, G_{k}$. Suppose $\Phi_{i}: G_{i} \times M \rightarrow M, i \in k$ are proper and free group actions on $M$. Then $D_{\Phi_{1}}, \ldots, D_{\Phi_{k}}$ are simultaneous integrable (i.e. all possible distributions $\sum_{i \in I} D_{\Phi_{i}}, I \in \underline{k}$ are involutive) if and only if for all $I \in \underline{k}, \bigcup_{i \in I} G_{i}$ is a closed Lie subgroup of $G$.

Proof. Immediate. 
We now come close to the construction of the group action on $G$. Suppose $G$ is given and we have proper and free group actions $\Phi_{i}: G_{i} \times M \rightarrow M, i=1,2$ of closed Lie subgroups $G_{i}$ of $G, i=1,2$.

Assume that $G_{1}$ and $G_{2}$ satisfy the conditions of proposition 4.8 . Then there exists a proper and free group action $\Phi:\left(G_{1} \cup G_{2}\right) \times M \rightarrow M$ if

$$
\left.\Phi_{1}\right|_{G_{1} \cap G_{2}}=\left.\Phi_{2}\right|_{G_{1} \cap G_{2}}
$$

Namely the closed Lie subgroup $G_{1} \cup G_{2}$ can be decomposed as

$$
G_{1} \cup G_{2}=\tilde{G}_{1} \oplus G_{1} \cap G_{2} \oplus \tilde{G}_{2} \text {, where } \tilde{G}_{1} \subset G_{1} \text { and } \tilde{G}_{2} \subset G_{2} .
$$

Given $g \in G_{1} \cup G_{2}$ we may write $g=g_{1} \cdot g_{12} \cdot g_{2}$ with $g_{1} \in \tilde{G}_{1}, g_{2} \in \tilde{G}_{2}$ and $g_{12} \in G_{1} \cap G_{2}$. Then $\Phi(g, \cdot)=\Phi_{1}\left(g_{1}, \Phi_{1}\left(g_{12}, \Phi_{2}\left(g_{2}, \cdot\right)\right)\right)$. One easily verifies that, although the decomposition (4.15) is not unique, the action is welldefined. Moreover $\Phi_{1}\left(g_{1}, \Phi_{1}\left(g_{12}, \Phi_{2}\left(g_{2}, \cdot\right)\right)\right)=\Phi_{1}\left(g_{1}, \Phi_{2}\left(g_{12}, \Phi_{2}\left(g_{2}, \cdot\right)\right)\right)=$ $\Phi_{2}\left(g_{2}, \Phi_{2}\left(g_{12}, \Phi_{1}\left(g_{1}, \cdot\right)\right)\right)$, where the last equality is proved analogously as in lemma 4.5. Therefore the order of the $\Phi_{i}$ 's in the definition of $\Phi$ may be reversed. Notice that $\Phi$ is uniquely defined on $G_{1} \cup G_{2}$. The foregoing discussion reveals whether or not a group action $\Phi$ can be defined on $\bigcup_{i \in k} G_{i}$, where the $G_{i}$ 's satisfy the conditions of proposition 4.8. We will call the actions $\Phi_{1}, \ldots, \Phi_{k}$ compatible if such an action exists. That is, firstly condition (4.15) should be satisfied for all $\left(G_{i}, \Phi_{i}\right)$ and $\left(G_{j}, \Phi_{j}\right), i, j \in k$. Then secondly while there is a unique action, say $\Phi^{i j}$ on $G_{i} \cup G_{j}$ the same condition (4.15) should hold for all $\left(G_{i} \cup G_{j}, \Phi^{i j}\right)$ and $\left(G_{l}, \Phi_{l}\right), i, j, l \in k$. The remaining conditions for compatibility of the $\Phi_{i}$ 's now should be obvious.

Proposition 4.8 and the above considerations may be summarized as follows

Lemma 4.9. Suppose $G_{1}, \ldots, G_{k}$ are closed Lie subgroups of a Lie group $G$. Let $\left(G_{1}, \Phi_{1}\right), \ldots,\left(G_{k}, \Phi_{k}\right)$ be proper and free Lie group actions on $M$. There exists $a$ proper and free group action $\Phi: G \times M \rightarrow M$ with $\left.\Phi\right|_{G_{i}}=\Phi_{i}$ if

1) for all $I \subset \underline{k}, \bigcup_{i \in I} G_{i}$ is a closed Lie subgroup of $G(\cup$ denotes the set theoretic union) and $\bigcup_{i \in k}^{i \in I} G_{i}=G$.

2) The group actions $\Phi_{1}, \ldots, \Phi_{k}$ are compatible.

Therefore, see lemma 4.2 and lemma 4.9 , we finally obtain:

Corollary 4.10. Suppose $\left(G_{1}, \Theta_{1}, \Phi_{1}\right), \ldots,\left(G_{k}, \Theta_{k}, \Phi_{k}\right)$ are partial symmetries for the affine system (4.1), and where $G_{1}, \ldots, G_{k}$ are closed Lie subgroups of a Lie group G. Suppose that

1) for all $I \subset \underline{k}, \bigcup_{i \in I} G_{i}$ is a closed Lie subgroup of $G$ and $\bigcup_{i \in k} G_{i}=G$.

2) The group actions $\Phi_{1}, \ldots, \Phi_{k}$ are compatible. Then there exists a partial symmetry $(G, \Theta, \Phi)$ for (4.1) with $\left.\Phi\right|_{G_{i}}=\Phi_{i}, i \in \underline{k}$.

Remarks. (i) A very easy similar sort of results may be obtained for systems which admit a triangular decomposition, see [9]. 
(ii) Most of the results of this section are also valid for a general nonlinear system $\Sigma(M, B, f)$. Basically one needs results on input-output decoupling for these systems. This will be reported in a forthcoming paper, [17].

\section{References}

1. R. Abraham and J. E. Marsden, Foundations of Mechanics, 2nd ed., Benjamin/Cummings Publishing Comp, London, 1978.

2. V. Arnold, Méthodes Mathématiques de la Méchanique Classique, MIR, Moscow, 1976.

3. J. W. Grizzle, The structure and optimization of nonlinear control systems possessing symmetries, Ph.D. thesis, University of Texas, 1983.

4. J. W. Grizzle and S. I. Marcus, The structure of nonlinear control systems possessing symmetries, to appear in $I E E E-T-A C$.

5. A. Isidori, A. J. Krener, C. Gori-Giorgi and S. Monaco, Nonlinear decoupling via feedback: a differential geometric approach, IEEE-T-AC, 26, 331-345, 1981.

6. A. Isidori, A. J. Krener, C. Gori-Giorgi, S. Monaco, Locally $(f, g)$-invariant distributions, Syst. Control Lett., 1, 12-15, 1981.

7. A. J. Krener, A decomposition theory for differentiable systems, SIAM J. Control and Optimiz., 15, 813-829, 1977.

8. H. Nijmeijer, Feedback decomposition of nonlinear control systems, IEEE-T-AC, 28, 861-862, 1983.

9. H. Nijmeijer, The triangular decoupling problem for nonlinear control systems, Nonlinear Analysis, Theory and Its Applic. 8, 273-279, 1984.

10. H. Nijmeijer and A. J. van der Schaft, Controlled invariance for nonlinear systems, $I E E E-T-A C$, 27, 904-914, 1982.

11. H. Nijmeijer and J. M. Schumacher, The noninteracting control problem for nonlinear control systems, Memo 427, Dept. Appl. Math., Technical University of Twente, 1983.

12. H. Nijmeijer and J. M. Schumacher, Zeros at infinity for affine nonlinear control systems, Memo 441, Dept. Appl. Math., Technical University of Twente, 1983.

13. W. Respondek, On decomposition of nonlinear control systems, Syst. Control Lett., 1, 301-308, 1982.

14. H. J. Sussmann and V. Jurdjevic, Controllability of nonlinear systems, J. Diff. Equat., 12, 95-116, 1972.

15. A. J. van der Schaft, Symmetries and conservation laws for Hamiltonian systems with inputs and outputs: A generalization of Noether's theorem, Syst. Control Lett, 1, 108-115, 1981.

16. A. J. van der Schaft, System theoretic descriptions of physical systems, Doctoral Dissertation, University of Groningen, 1983, and CWI Tracts No. 3, Amsterdam, 1984.

17. A. J. van der Schaft, Linearization and input-output decoupling for general nonlinear systems, to appear in Syst. Control Lett.

Received on February 22, 1984, and in revised form on May 15, 1984. 